case report

\title{
Mediastinitis and bilateral pleural empyema caused by an odontogenic infection
}

\author{
Mirna Juretic ${ }^{1}$, Margita Belusic-Gobic ${ }^{1}$, Melita Kukuljan ${ }^{3}$, Robert Cerovic ${ }^{1}$, \\ Vesna Golubovic ${ }^{2}$, David Gobic ${ }^{4}$ \\ ${ }^{1}$ Clinic for Oral and Maxillofacial Surgery, ${ }^{2}$ Clinic for Anaesthesiology and Reanimatology, \\ ${ }^{3}$ Department of Radiology, ${ }^{4}$ Clinic for Internal Medicine, Clinical hospital, Rijeka, Croatia
}

\begin{abstract}
Background. Although odontogenic infections are relatively frequent in the general population, intrathoracic dissemination is a rare complication. Acute purulent mediastinitis, known as descending necrotizing mediastinitis (DNM), causes high mortality rate, even up to $40 \%$, despite high efficacy of antibiotic therapy and surgical interventions. In rare cases, unilateral or bilateral pleural empyema develops as a complication of DNM.

Case report. This case report presents the treatment of a young, previously healthy patient with mediastinitis and bilateral pleural empyema caused by an odontogenic infection. After a neck and pharynx re-incision, and as CT confirmed propagation of the abscess to the thorax, thoracotomy was performed followed by CT-controlled thoracic drainage with continued antibiotic therapy. The patient was cured, although the recognition of these complications was relatively delayed.

Conclusions. Early diagnosis of DNM can save the patient, so if this severe complication is suspected, thoracic CT should be performed.
\end{abstract}

Key words: mediastinitis; empyema, pleural; periapical abscess - complications

\section{Introduction}

Acute suppurative mediastinitis is a lifethreatening infection infrequently occurring as a result of the propagation of odontogenic infection, and is described as descending necrotizing mediastinitis (DNM). Pleural empyema is reported as a

Received 25 May 2007

Accepted 18 June 2007

Correspondence to: Belusic-Gobic Margita, MD, KBC Rijeka, Department of Oral and Maxillofacial Surgery, Tome Strižića 3, 51000 Rijeka, Croatia; Phone/Fax: +385 51218279; E-mail: mfk@kbc-rijeka.hr rare complication of acute mediastinitis. ${ }^{1-6}$ Clinical manifestations of mediastinitis are frequently nonspecific. If the diagnosis of mediastinitis is suspected, thoracic CT is required regardless of negative chest X-ray. In the treatment of advanced mediastinitis, administration of antibiotic therapy alone is ineffective unless aggressive surgical interventions including wide incisions, debridement, excision of necrotic tissue and drainage of all abscessed regions are included.

A case of a young patient with mediastinitis and bilateral pleural empyema caused by odontogenic infection is presented. 


\section{Case report}

A previously healthy 23-year-old man presented to his dentist complaining of toothache in the region of the right mandibular third molar. Root trepanation was performed and antibiotic therapy (amoxicillin) was initiated but the patient's condition worsened with severe pain and marked swelling. On the $9^{\text {th }}$ day after the occurrence of toothache, the patient was referred to the Clinic of maxillofacial and oral surgery. Physical examination revealed trismus, a firm swelling in the right submandibular and in a portion of supramandibular regions, oedema and erythema of retromolar mucosa. Due to an extensive dental caries the crown of the tooth was completely destroyed. The patient was subfebrile to $37.5{ }^{0} \mathrm{C}$, had dysphagia and slightly muffled voice. On admission to hospital, initial preoperative procedures were performed. Laboratory tests showed signs of inflammation: (WBC $12.6 \times 10^{9}$ / L, fibrinogen $9.09 \mathrm{~g} / \mathrm{l}$, CRP $18.9 \mathrm{mg} / \mathrm{dL})$. Chest $\mathrm{X}$-ray revealed no irregularities. The same evening under general endotracheal anaesthesia external incision was done by opening the submandibular triangle to the mandibular periost and to the buccal region, where purulent content was found to be less than expected. A larger amount of pus was obtained by a wide incision in the retromolar space. Ciprofloxacin 400 $\mathrm{mg} / 12 \mathrm{~h}$ i.v. and metronidazole $500 \mathrm{mg} / 8 \mathrm{~h}$ were prescribed till the results of cultures were obtained. On the 1st postoperative day the patient's condition improved subjectively, but he continued to be subfebrile, had dysphagia accompanied by sore throat and suppurative spit. Due to his private problems, he insisted on being discharged that same day. Four days later, the patient returned with worsened general condition and local findings. He complained of severe buccal and retromolar pressure, had severe dysphagia and a muffled voice («hot potato voice»), productive cough, and temperature of $37.8^{\circ} \mathrm{C}$. Clinical examination of the neck showed an infected induration also in the parotid region of the same side. Chest X-ray was again normal. Laboratory tests revealed further increase of leucocytes $\left(25.0 \times 10^{9} / \mathrm{L}\right)$ and CRP $(28.2$ $\mathrm{mg} / \mathrm{dL}$ ). The HIV test was negative. On the same day re-incision was performed extensively exploring the submandibultar triangle, parotid region, retromolar, retromandibular and pharyngeal spaces, and the infection-causing root of the lower third molar was extracted. The culture of the pus content indicated the presence of Streptococcus viridans and based on the antibiogram cefepime $2 \mathrm{~g} / 12 \mathrm{~h}$ i.v. was given.

Two days after the second incision, temperature raised to $38.3^{\circ} \mathrm{C}$ with severe pain below right rib arch, major dysphagia and cough. Neck ultrasound and CT were performed then which showed a large formation of thick fluid content and gas distal from the a. carotis bifurcation bilaterally. The thoracic CT scan revealed equal pathological formation in the mediastinum (Figures 1, 2).

Emergency posterolateral left thoracotomy through the fifth intercostal space was performed and about $550 \mathrm{~cm}^{3}$ of purulent material from the mediastinum was evacuated. Drains were placed, one in each of the three compartments of the mediastinum. During the same procedure wide incisions of the neck bilaterally were carried out, including the carotid spaces, suprasternal, parotid and parapharyngeal regions. Wide drains were placed on the neck bilaterally. The patient was transferred to the intensive care unit and attached to mechanical ventilation. Thromboprophylactic therapy was introduced and based on the cultures, $1 \mathrm{~g} / 12 \mathrm{~h}+$ amicacin sulphate $500 \mathrm{mg} / 12$ $\mathrm{h}$ were given. In a few postoperative days, 


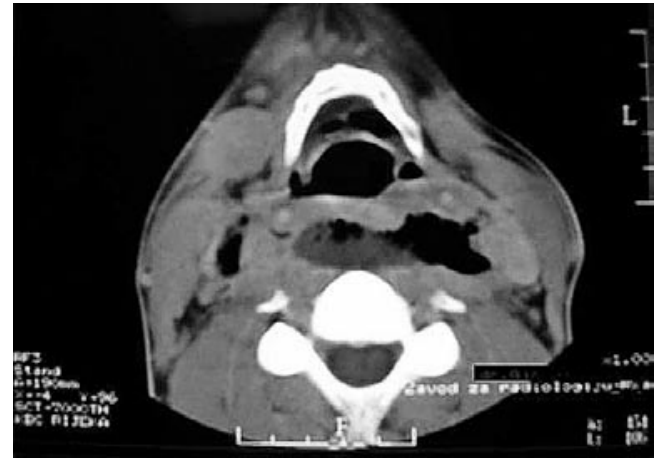

Figure 1. CT of the neck: fluid and gas collections in the retropharingeal compartments.

the patient was cardiocirculatory instable, with abnormal coagulogram, haematogram and hepatogram. Daily follow-up chest Xrays were performed, and on the 7 th day after thoracotomy the follow-up chest X-ray showed lower transparency of lung lobes, indicating a follow-up thoracic CT was required. The CT scan demonstrated enormous bilateral pleural empyemas, with a marked reduction in respiratory space and a paracardial purulence (Figure 3).

Due to extremely worsened general condition of the patient, percutaneous bilateral thoracic CT-controlled drainage was performed, $1800 \mathrm{ml}$ of pus was evacuated

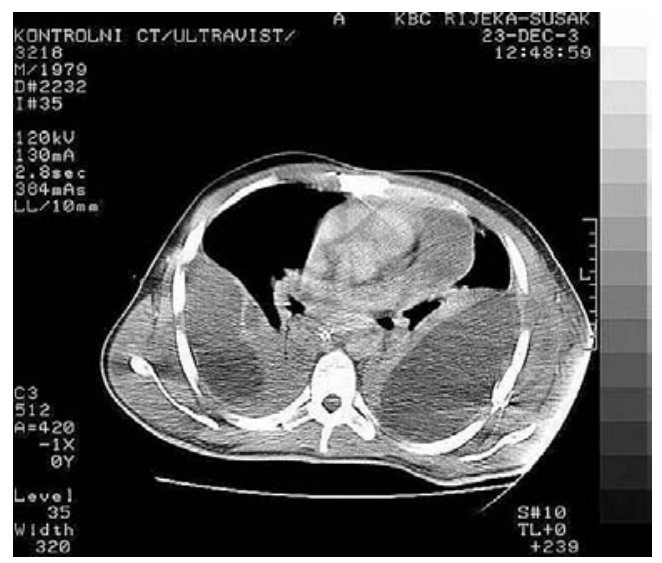

Figure 3. CT of the thorax: bilateral pleural empyema.

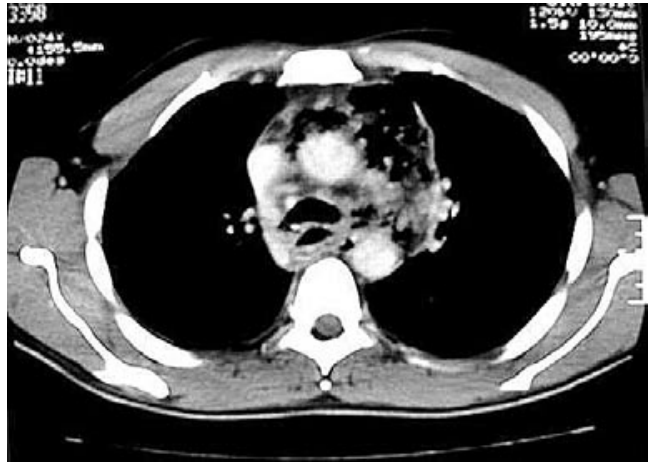

Figure 2. CT of the mediastinum: mediastianal purulence and clear lungs.

and a significant lung re-expansion was obtained. A suction drain was placed in each chest (Figure 4).

Antibiotic therapy was changed to vancomycin $1 \mathrm{~g} / 12 \mathrm{~h}+$ metronidazole $500 \mathrm{mg} / 8 \mathrm{~h}$ (based on the cultures of purulent material obtained by drains). The patient's condition gradually improved. On the 22nd day after percutaneous drainage, chest drains were removed, and on the 25th day mechanical ventilation was removed. The patient was discharged from the intensive care unit after the total stay of 37 days, without any significant consequences except slightly muffled voice.

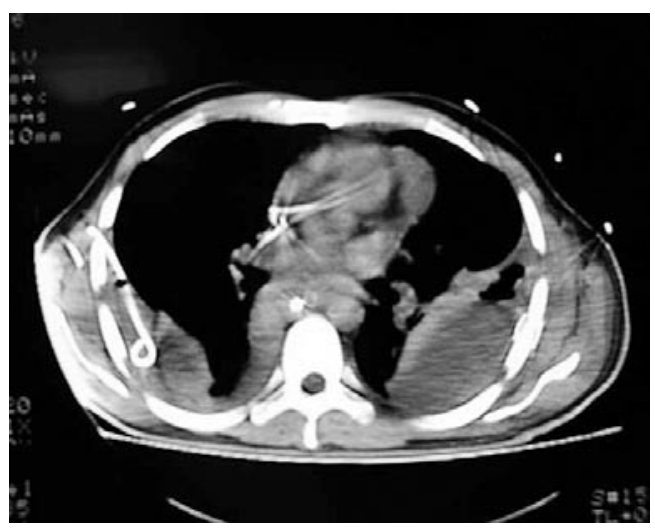

Figure 4. CT of the thorax after drainage: bilateral drains in the pleural space. 


\section{Discussion}

This case demonstrates potentially disastrous effects of odontogenic infections. DNM is a rare result of a dental infection, and even more infrequently, bilateral pleural empyema develops as a complication of DNM. $^{1,3-7}$ In the period 1990-2004, there were no cases of mediastinitis after orodental or oropharyngeal infection in our epidemiologic centre which covers the population of about 400,000 people. Either due to a long period without serious complications of odontogenic infections (although an average of 50 patients with odontogenic abscess are admitted to hospital annually), or because the young man was previously completely healthy without a history of any disease or addiction, and palpation of the neck did not show a larger induration and relatively low fever considering the severity of the condition, this complication was diagnosed with a delay, which sometimes contributes to a high mortality rate of DNM. Estrere et al. report that one third of all cases of mediastinitis are diagnosed at post-mortem examination. ${ }^{8}$ Symptoms of cough, dysphagia and dyspnoea, swelling of the neck and upper part of the chest with crepitance indicate clinical manifestations of mediastinitis, but these are late signs. Early diagnosis requires radiologic evaluation and CT is indispensable because clinical examination alone is reliable in only $55 \%$ of cases. Besides, CT is beneficial in

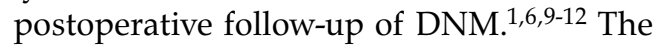
fact is that a delay in diagnosis of several days results in severe complications such as pyothorax, aortopulmonal fistula, sepsis, the erosion of the carotid artery or aorta, purulent pericarditis, DIC and multiple damages to the organs. ${ }^{13}$ Our patient developed bilateral pleural empyema which is infrequently described as a complication of odontogenic DNM. ${ }^{1,-5,7}$ Inadequate surgical drainage can lead to deterioration of the patient's general condition and can cause new infectious foci in residual abscesses. Therefore, the meticulous drainage of the abscess, coupled with antibiotics and $\gamma$ globulin as supportive therapy. ${ }^{13,14}$ is mandatory. For minor infections, most authors recommend penicillin and metronidazole per os for 7 days as the first- line therapy in out-patients. For major infections, authors suggest penicillin $\mathrm{G} 4$ million units every 6 h i.v. together with metronidazole $500 \mathrm{mg}$ every $8 \mathrm{~h}$ i.v., and against gram- negative species additional gentamicin $3 \mathrm{mg} / \mathrm{kg}$ i.v. every 24 h. ${ }^{15,16,17}$ Maisel and Karlen recommend simultaneous administration of the third generation of cephalosporin and clindamycin or metronidazole as initial antimicrobial coverage until the results of the antibiogram are obtained. ${ }^{18}$

The approach to the drainage of mediastinum remains a moot point. Nevertheless, in case of a massive infection transthoracic approach is required. ${ }^{1,6,8,10,12,13,19}$ Posterolateral thoracotomy provides adequate drainage of all portions of the mediastinum, pleural cavity and pericardium. One of the issues is also to perform tracheostomy immediately or to leave a tracheal tube. Our patient had a tube during the whole treatment with mechanical ventilation. Many authors prefer immediate tracheostomy in major cervical infections, to prevent the risk of additional intubations after accidental dislodgement of endotracheal tube in head movements. Other authors prefer intubation in all cases without respiratory impairment since opening of the airway by tracheostomy can result in the propagation of infection to the lungs. Opening cervical fascia in tracheostomy exposes the patient to the contamination of pretracheal space and to the risk of caudal spread of the infection to the mediastinum. ${ }^{6,8,12,19-22}$

According to the literature cervical infections are more common in immunosuppressive patients ${ }^{8}$ but Mathieu at al. ${ }^{23}$ re- 
ported that $58 \%$ of cases necrotizing fascitis develop in previously healthy patients like in described case.

Although DNM as a complication of dental infection is thought to be an infrequent clinical entity, according to Sakamoto et al., DNM strictly related to oral causes accounts for $60-70 \%$ of all reported cases. ${ }^{22}$ Usually it originates in the second or third mandibular molar, which roots lie below the mylohyoid muscle providing the infection with a possibility of an immediate access to the submandibular space. ${ }^{8,24,25}$ In our patient, the third molar was involved.

With a view to all this, in the case of the patient reported here, there were several pointers which indicated the possibility of this complication. Out- patient treatment in the dental office took some time with questionable need for trepanation of less valuable residual root of the third molar. Even on admission of the patient, the presenting clinical signs and laboratory findings should be followed by CT scan, which was delayed due to underestimation of the patient's condition, although he was eventually successfully managed.

\section{Conclusions}

Early diagnosis, aggressive drainage and antibiotic therapy with adequate postoperative care can save a patient with DNM, despite a high mortality rate of this disease.

Therefore, practitioners who face cases of odontogenic infection must be aware of this severe complication even in previously healthy patients, and together with adequate knowledge of this entity, must be able to recognize prodromal symptoms of the disease and if DNM is suspected to perform CT examination.

\section{References}

1. Balkan ME, Oktar GL, Oktar MA. Descending necrotizing mediastinitis: a case report and review of the literature. Int Surg 2001; 86: 62-6.

2. Izadi K, Lazow SK, Berger JR. Mediastinitis secondary to an odontogenic infection. A case report. N Y State Dent J 2003; 69: 28-30.

3. Tung-Yiu W, Jehn-Shyun H, Ching-Hung C, HungAn C. Cervical necrotizing fasciitis of odontogenic origin: a report of 11 cases. J Oral Maxillofac Surg 2000; 58: 1347-53.

4. Sobolewska E, Skokowski J, Jadezuk E. Pleural empyema as a complication of the descending necrotizing mediastinitis. Pneumonol Alergol Pol 1997; 65: 364-9.

5. Bonapart IE, Stevens HP, Kerver AJ, Rietveld AP. Rare complications of an odontogenic abscess: mediastinitis, thoracic empyema and cardiac tamponade. J Oral Maxillofac Surg 1995; 53: 610-3.

6. Biasotto $\mathrm{M}$, Pellis $\mathrm{T}$, Cadenaro $\mathrm{M}$, Bevilacqua $\mathrm{L}$, Berlot G, Di Leonarda R. Odontogenic infections and descending mediastinitis: case report and review of the literature. Int Dent J 2004; 54: 97-102.

7. Economopoulos GC, Scherzer HH, GryboskiWA. Successful management of mediastinitis, pleural empyema, and aortopulmonary fistula from odontogenic infection. Ann Thorac Surg 1983; 35: 184-7.

8. Estrera AS, Landay MJ, Grisham JM, Sinn DP, Platt MR. Descending necrotizing mediastinitis. Surg Gynecol Obstet 1983; 157: 545-52.

9. Miller WD, Furst IM, Sandor GK, Keller A. A prospective blinded comparison of clinical exam and computed tomography in deep neck infections. Laryngoscope 1999; 109: 1873-9.

10. Tsunoda R, Suda S, Fukaya T, Saito K. Descending necrotizing mediastinitis caused by an odontogenic infection: a case report. J Oral Maxillofac Surg 2000; 58: 240-2.

11. Garcia-Consuegra L, Junquera-Gutierrez L, Albertos-Castro JM, Llorente-Pendas S. Descending necrootizing mediastinitis caused by odontogenic infections. Rev Stomatol Chir Maxillofac 1998; 99: 199-202.

12. Marty-Ane $\mathrm{CH}$, Alauzen $\mathrm{M}$, Alric $\mathrm{P}$, Serres-Cousine $\mathrm{O}$, Mary $\mathrm{H}$. Descending necrotizing mediastinitis: avantage of mediastinal drainage with thoracotomy. J Thorac Cardiovasc Surg 1994; 107: 55-61. 
13. Iyoda A, Yusa T, Fujisawa T, Mabashi T, Hiroshima $\mathrm{K}$, Ohwada $\mathrm{H}$. Descending necrotizing mediastinitis: report of a case. Surg Today 1999; 29: 1209-12.

14. Labriola JD, Mascaro J, Alpert B. The microbiologic flora of orofacial abscesses. J Oral Maxillofac Surg 1983; 41: 711-4.

15. Pynn BR, Sands T, Pharoah MJ. Odontogenic infections: Part one. Anatomy and radiology. Oral Health 1995; 85: 7-10, 13-4, 17-8.

16. Sands T, Pynn BR, Katsikeris N. Odontogenic infections: Part two. Microbiology, antibiotics and management. Oral Health 1995; 85: 11-4, 17-23.

17. Sandor GK, Low DE, Judd PL, Davidson RJ. Antimicrobial treatment options in the management of odontogenic infections. J Can Dent Assoc 1998; 64: 508-14.

18. Maisel RH, Karlen R. Cervical necrotising fascitis. Laryngoscope 1994; 104: 795-8.

19. Wheatley MJ, Stirling MC, Kirsh MM, Gago O, Orringer MB. Descending necrotizing mediastinitis: transcervical drainage is not enough. Ann Thorac Surg 1990; 49: 780-4.

20. Brunelli A, Sabbatini A, Catalini G, Fianchini A. Descending necrotizing mediastinitis. Surgical drainage and tracheostomy. Arch Otolaryngol Head Neck Surg 1996; 122: 1326-9.

21. Cordero L, Torre W, Freire D. Descending necrotizing mediastinitis and respiratory distress syndrome treated by aggressive surgical treatment. $J$ Cardiovasc Surg 1996; 37: 87-8.

22. Sakamoto H, Aoki T, Kise Y, Watanabe D, Sasaki J. Descending necrotising mediastinitis due to odontogenic infections. Oral Surg Oral Med Oral Path Radiol Endod 2000; 89: 412-9.

23. Mathieu D, Neviere R, Teillon C, Chagnon JL, Lebleu N, Wattel F. Cervical necrotizing fascitis: clinical manifestations and management. Clin Infect Dis 1995; 21: 51-6.

24. Sugata T, Fujita Y, Myoken Y, Fujioka Y. Cervical cellulitis with mediastinitis from an odontogenic infection complicated by diabetes mellitus: report of a case. J Oral Maxillofac Surg 1997; 55: 864-9.

25. Rubin MM, Cozzi GM. Fatal necrotizing mediastinitis as a complication of an odontogenic infection. J Oral Maxillofac Surg 1987; 45: 529-33. 